\title{
Immunohistochemical Reactivity of Prostate-Specific Markers for Salivary Duct Carcinoma
}

\author{
Nami Takada ${ }^{a}$ Haruto Nishida ${ }^{a}$ Yuzo Oyama $^{a}$ Takahiro Kusaba ${ }^{a}$ \\ Hiroko Kadowaki $^{a}$ Motoki Arakane ${ }^{a}$ Junpei Wada ${ }^{b}$ Shogo Urabe ${ }^{b}$ \\ Tsutomu Daa ${ }^{a}$ \\ ${ }^{a}$ Department of Diagnostic Pathology, Faculty of Medicine, Oita University, Oita, Japan; \\ ${ }^{b}$ Department of Pathology, Clinical Laboratory, Oita Prefectural Hospital, Oita, Japan
}

\section{Keywords}

Salivary duct carcinoma - NKX3.1 - a-Methylacyl-CoA racemase $\cdot$ Prostatic acid phosphatase $\cdot$ Prognosis

\begin{abstract}
Objectives: NKX3.1, a transcription factor related to androgen expression, has recently been introduced as a diagnostic marker of prostate adenocarcinoma. Salivary duct carcino$\mathrm{ma}(\mathrm{SDC})$ is typically positive for androgen receptor (AR). Therefore, we hypothesized that NKX3.1 is a new immunohistochemical marker for SDC and aimed to investigate whether NKX3.1 staining in combination with other immunomarkers of prostate carcinoma could have a diagnostic or prognostic value in SDC. Methods: Materials obtained from 42 resected SDCs were examined by immunohistochemistry using antibodies against AR, NKX3.1, a-methylacyl-CoA racemase (AMACR), prostatic acid phosphatase (PAP), and prostate-specific antigen (PSA). Results: In immunoreactivity among SDC cases, 81.0, 35.7, 58.5, 33.3, and $0 \%$ were positive for AR, NKX3.1, AMACR, PAP, and PSA, respectively. AMACR and $\mathrm{PAP}$ immunoreactivity rates were higher in recurrence
\end{abstract}

karger@karger.com

(c) 2019 S. Karger AG, Basel

www.karger.com/pat

Karger" cases than in cases with no recurrence. Conclusions: NKX3.1 expression is useful for SDC diagnosis, but decreased NKX3.1 expression was not correlated with SDC progression. The immunoreactivity of AMACR and PAP could be useful for assessing prognosis in SDC, but immunohistochemical staining of prostate-specific markers should be interpreted with caution when determining whether a metastatic tumor is of prostate origin, especially when patients have a history of SDC.

(c) 2019 S. Karger AG, Basel

\section{Introduction}

Salivary duct carcinoma (SDC) is a relatively rare salivary gland malignancy [1-3]. Its histological characteristics are generally similar to those of invasive ductal carcinoma of the breast [1-3]. Most cases occur de novo, although some represent the malignant component of carcinoma ex pleomorphic adenoma (PA). Immunohistochemically, SDCs are typically positive for androgen receptor (AR), which serves as a confirmative diagnostic 
Table 1. Antigen retrieval methods and primary antibodies used in immunostaining

\begin{tabular}{lllll}
\hline Antibody & Antigen retrieval & Clonality (clone) & Vendor & Location \\
\hline AR & AC $(\mathrm{pH} \mathrm{6)}$ & Monoclonal (AR441) & Dako & Glostrup, Denmark \\
NKX3.1 & AC $(\mathrm{pH} \mathrm{6)}$ & Monoclonal (A02709*) & Boster & Pleasanton, CA, USA \\
AMACR & HIER $(\mathrm{pH} \mathrm{6)}$ & Monoclonal (N/A) & Biocare Medical & Pacheco, CA, USA \\
PAP & - & Polyclonal & Dako & Glostrup, Denmark \\
PSA & - & Polyclonal & Dako & Glostrup, Denmark \\
\hline
\end{tabular}

* Catalog number. AC, autoclave; HIER, heat-induced epitope retrieval; AR, androgen receptor; AMACR, $\alpha$-methylacyl-CoA racemase; PAP, prostatic acid phosphatase; PSA, prostate-specific antigen.

Table 2. Clinical feature of salivary duct carcinoma

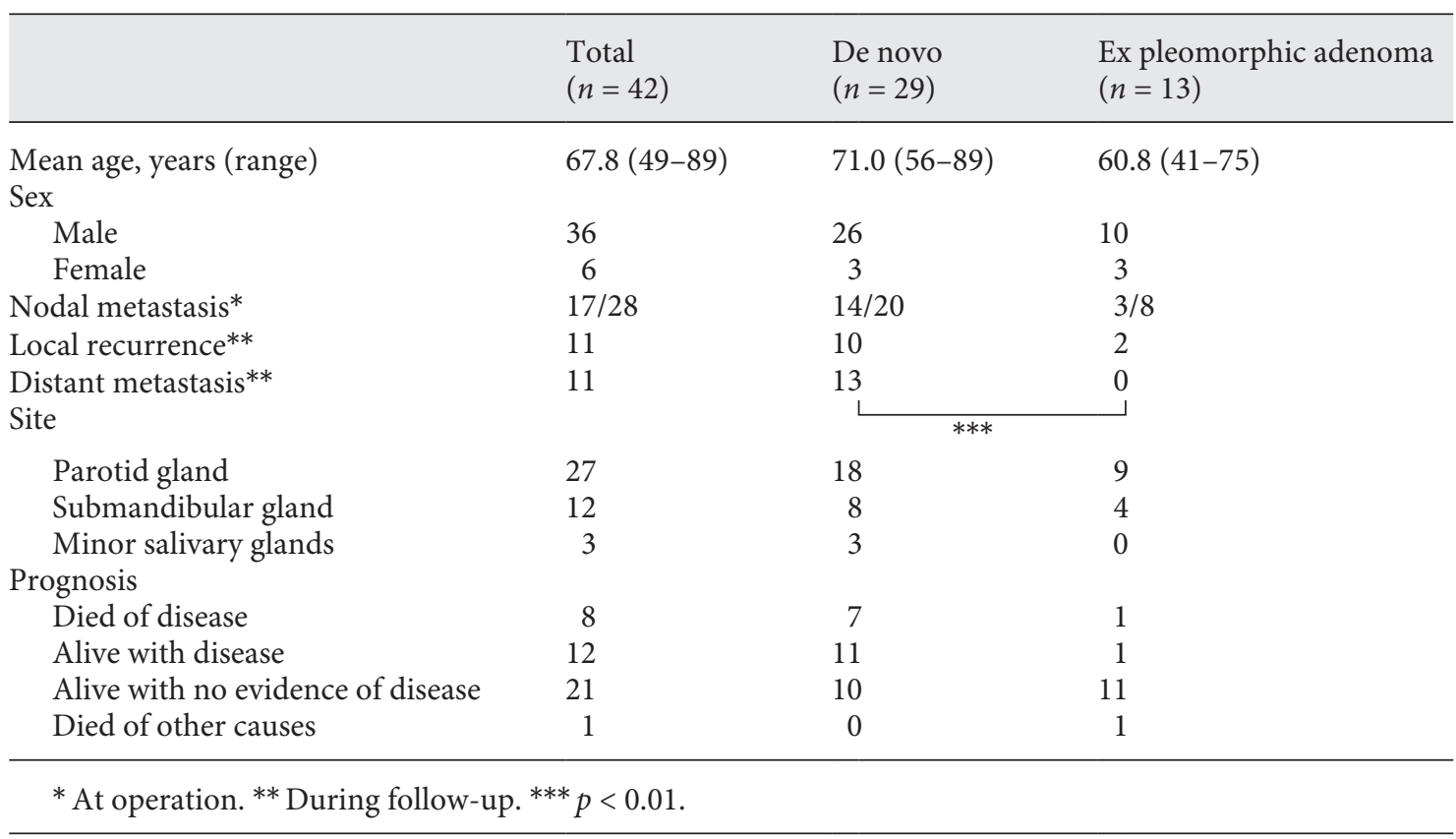

marker [4]. AR plays essential roles in the development and progression of prostate cancer $[5,6]$. A recent study has shown that immunohistochemistry staining NKX3.1 has diagnostic value in prostatic adenocarcinomas; this marker is related to androgen expression [7]. NKX3.1 is an androgen-regulated homeodomain gene whose expression is predominantly localized to the prostate epithelium [7]. According to previous studies, NKX3.1 expression is significantly decreased in prostate cancer patients compared to those with benign prostate hyperplasia [8]. Further, loss of NKX3.1 expression is strongly associated with hormone-refractory disease and advanced tumor stage in prostate cancer [9]. We hypothesized that NKX3.1 is a new immunohistochemical marker for SDC; however, to the best of our knowledge, few studies have examined this prediction [10]. Therefore, the purpose of this study was to investigate whether NKX3.1 staining in combination with other immunomarkers of prostate carcinoma could have a diagnostic and prognostic value in SDC.

\section{Materials and Methods}

In total, 42 SDCs were resected at Oita University and Oita Prefectural Hospital between February 2004 and June 2018. The patients included 36 men and 6 women, with a mean age of 67.8 years (range, 49-89 years). The primary sites of SDCs were the parotid gland in 27 cases, the submandibular gland in 12 cases, and the 


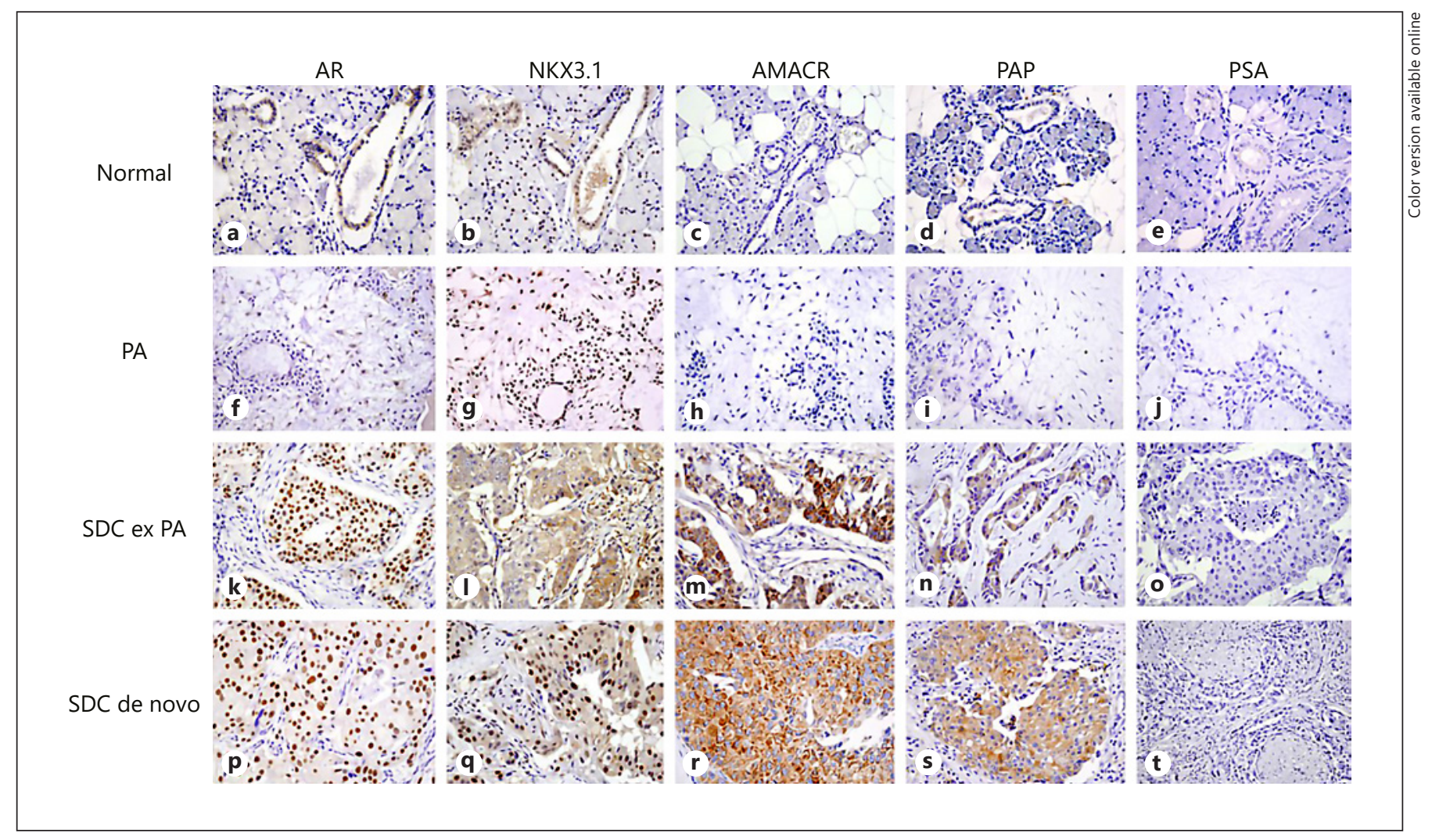

Fig. 1. Immunohistochemical findings for AR, NKX3.1, AMACR, PAP, and PSA in normal salivary gland (a-e), pleomorphic adenoma (PA) (f-j), salivary duct carcinoma (SDC) ex PA (k-o), and de novo SDC (p-t). NKX3.1 and AR were expressed in the nuclei, and AMACR, PAP, and PSA were detected in the cytoplasm. With respect to immunoreactivity in normal salivary glands, serous acini and ducts showed positivity for NKX3.1, whereas AR, AMACR, PAP, and PSA were negative. The representative case of PA shows

minor salivary glands in 3 cases. Histological features were assessed using hematoxylin-eosin-stained sections from formalinfixed, paraffin-embedded tissues by N.T. and H.N. For immunohistochemical analyses, representative tissue blocks were selected. Immunohistochemical staining was performed manually. Primary antibodies and antigen retrieval methods for immunostaining are indicated in Table 1. The sections were deparaffinized in xylene and rehydrated in a graded ethanol series. Endogenous peroxidase activity was abolished by incubation with $3 \%$ hydrogen peroxide for $20 \mathrm{~min}$ at room temperature. The antigens were retrieved according to the manufacturer's instructions. The slides were incubated with the first antibody and stained using the streptavidinlabeled biotin peroxidase complex system (Nichirei, Tokyo, Japan). They were visualized using diaminobenzidine tetrahydrochloride (DAB) solution. More than 30\% reactivity in tumor cells and higher staining intensity than that of normal ductal or acinar cells indicated a positive result. Differences were analyzed using Fisher's probability test; $p<0.05$ was considered statistically significant. nuclear positivity for NKX3.1 (g), and the others were negative in the epithelial and myoepithelial component. The representative case of SDC ex PA shows nuclear positivity for AR (k) and cytoplasmic positivity for AMACR $(\mathbf{m})$ and $\mathrm{PAP}(\mathbf{n})$, but was negative for NKX3.1 (I) and PSA (o). The representative case of de novo SDC shows nuclear positivity for AR (p) and NKX3.1 (q) and cytoplasmic positivity for AMACR (r) and PAP (s), but was negative for PSA (t).

\section{Results}

The clinical findings for SDC are summarized in Table 2. Of the 42 cases diagnosed as SDC, 29 (69.0\%) cases were de novo SDC and 13 cases (31.0\%) were SDC ex PA. Both types were most common in the parotid gland and in elderly men. Nodal metastasis was observed in 17 $(60.7 \%)$ of 28 cases with lymph node resection. A total of $14(70.0 \%)$ of 20 de novo SDC cases with lymph node resection showed nodal metastasis, while $3(37.5 \%)$ of $8 \mathrm{ex}$ PA cases showed nodal metastasis $(p=0.123)$. Eight (19.0\%) patients died of the disease, with a mean survival of 17 months after diagnosis (range, 2-28 months), and 7 cases $(87.5 \%)$ of these 8 cases were de novo. Regarding the histological findings of SDC ex PA, 7 of 13 cases were intracapsular carcinoma. The remaining 6 cases included 3 
Table 3. Immunocytochemistry analysis of the SDC with de novo and ex PA, and PA

\begin{tabular}{clllll}
\hline & AR & NKX3.1 & AMACR & PAP & PSA \\
\hline SDC $(n=42)$ & $81.0 \%(34 / 42)$ & $35.7 \%(15 / 42)$ & $58.5 \%(24 / 41)$ & $33.3 \%(14 / 42)$ & $0 \%(0 / 42)$ \\
De novo $(n=29)$ & $88.9 \%(24 / 27)$ & $37.9 \%(11 / 29)$ & $60.7 \%(17 / 28)$ & $37.9 \%(11 / 29)$ & $0 \%(0 / 29)$ \\
Ex PA $(n=13)$ & $76.9 \%(10 / 13)$ & $30.8 \%(4 / 13)$ & $53.8 \%(7 / 13)$ & $23.1 \%(3 / 13)$ & $0 \%(0 / 13)$ \\
PA $^{*}(n=13)$ & $23.0 \%(3 / 13)$ & $92.3 \%(12 / 13)$ & $15.4 \%(2 / 13)$ & $0 \%(0 / 13)$ & $0 \%(0 / 13)$ \\
\hline
\end{tabular}

* PA area in SDC ex PA. SDC, salivary duct carcinoma; PA, pleomorphic adenoma; AR, androgen receptor; AMACR, $a-$ methylacylCoA racemase; PAP, prostatic acid phosphatase; PSA, prostate-specific antigen.

Table 4. Comparing immunohistochemical staining in local recurrence and distant metastasis cases during follow-up

\begin{tabular}{|c|c|c|c|c|c|c|}
\hline Local recurrence $(n=12)$ & $90.9 \%(10 / 11)$ & $33.3 \%(4 / 12)$ & $83.3 \%(10 / 12)$ & & $41.7 \%(5 / 12)$ & $0 \%(0 / 12)$ \\
\hline Ex PA (2) & $100 \%(2 / 2)$ & $100 \%(2 / 2)$ & $100 \%(2 / 2)$ & & $100 \%(2 / 2)$ & $0 \%(0 / 2)$ \\
\hline Distant metastasis $(n=13)$ & $91.7 \%(11 / 12)$ & $30.8 \%(4 / 13)$ & $66.7 \%(8 / 12)$ & & $50.0 \%(6 / 12)$ & $0 \%(0 / 13)$ \\
\hline De novo (13) & $91.7 \%(11 / 12)$ & $30.8 \%(4 / 13)$ & $66.7 \%(8 / 12)$ & & $50.0 \%(6 / 12)$ & $0 \%(0 / 13)$ \\
\hline De novo (11) & $90 \%(9 / 10)$ & $63.6 \%(7 / 11)$ & $45.4 \%(5 / 11)$ & & $36.3 \%(4 / 11)$ & $0 \%(0 / 11)$ \\
\hline Ex PA (11) & $72.7 \%(8 / 11)$ & $18.2 \%(2 / 11)$ & $45.4 \%(5 / 11)$ & & $9.0 \%(1 / 11)$ & $0 \%(0 / 11)$ \\
\hline
\end{tabular}

* Cases with no local recurrence or distant metastasis during follow-up. ${ }^{* *} p<0.05$. AR, androgen receptor; AMACR, $a-m e t h y l a c y l-$ CoA racemase; PAP, prostatic acid phosphatase; PSA, prostate-specific antigen; PA, pleomorphic adenoma.

minimally invasive carcinomas and 3 invasive carcinomas. Eleven cases ( 7 intracapsular carcinomas, 2 minimally invasive carcinomas, and 2 invasive carcinoma) were non-recurrence cases. Two cases were locally recurrent ( 1 intracapsular carcinoma and 1 invasive carcino$\mathrm{ma})$, of which the latter died of the disease. No significant differences were noted in terms of age, sex, regional nodal metastasis at operation, or recurrence during follow-up between the two clinicopathologic forms, but distant metastasis during follow-up showed a significant difference $(p<0.0027)$.

The representative panel of the immunoreactivity of prostate carcinoma markers in normal salivary gland, SDC ex PA, and de novo SDC is shown in Figure 1. The results of immunohistochemical analysis for the $\mathrm{PA}$ area in SDC ex PA, SDC de novo, and SDC ex PA are presented in Table 3. NKX3.1 and AR were expressed in the nuclei, and $\alpha$-methylacyl-CoA racemase (AMACR), prostatic acid phosphatase (PAP), and prostate-specific antigen (PSA) were detected in the cytoplasm. With respect to immunoreactivity in normal salivary glands, serous acini (mucinous acini were negative) and ducts showed

Immunomarker Expression in Salivary

Duct Carcinoma positivity for NKX3.1. AR, AMACR, PAP, and PSA were negative in normal samples. In the immunoreactivity of the PA area in SDC ex PA cases, epithelial and myoepithelial components showed positivity for NKX3.1 (92.3\%), whereas AR and AMACR tended to show weak positivity compared with the SDC area. The AR-positive cases only showed positivity in the epithelial component. PAP and PSA were negative in the PA area. Among NKX3.1-positive PA cases, 12 out of 13 were negative for NKX3.1 in the SDC area. AR- and AMACR-positive PA cases were also positive in the SDC area.

Among all SDC cases, 81.0, 35.7, 58.5, 33.3\%, and 0\% were positive for AR, NKX3.1, AMACR, PAP, and PSA, respectively. The incidences of these marker-positive de novo SDC cases were higher than those of SDC ex PA cases, although the difference was not statistically significant. Comparisons of immunohistochemical staining results for cases with local recurrence and distant metastasis during follow-up are shown in Table 4. The cases were divided into three groups, namely, cases with local recurrence, distant metastasis, and no local recurrence or distant metastasis (no recurrence). In total, $33.3 \%$ of cases 
with local recurrence, $30.8 \%$ of cases with distant metastasis, and $40.9 \%$ of cases with no recurrence were positive for NKX3.1. The positive rates for AMACR of the local recurrence, distant metastasis, and no recurrence groups were $83.3,66.7$, and $45.4 \%$, respectively, and the differences were significant $(p<0.05$; local recurrence: $p=$ 0.0352 , distant metastasis: $p=0.0205)$. Similarly, with respect to positivity for PAP, cases with no recurrence (22.7\%) were less frequent than those with local recurrence (41.7\%) and distant metastasis (50.0\%). There were no significant differences in the immunoreactivity of AR, NKX3.1, AMACR, PAP, and PSA between de novo SDC and SDC ex PA, including intracapsular, minimally invasive, and invasive carcinoma, and between invasive and noninvasive areas.

\section{Discussion}

NKX3.1, located on chromosome 8p21.2, is an androgen-regulated homeodomain gene that is predominantly expressed in the prostate epithelium $[7,11]$. NKX3.1 directly regulates AR-dependent genes, which are overexpressed in prostate carcinoma, deregulated in advanced prostate cancer, and enriched in recurrent prostate cancer [7]. Moreover, NKX3.1 likely regulates genes that are active in promoting cell survival or preventing cell apoptosis [11]. Considering this, it is likely that NKX3.1 plays a pivotal role in prostate tumorigenicity. Using immunohistochemistry, Irer et al. [12] reported that NKX3.1 expression was elevated in benign prostate hyperplasia tissues compared with that in normal tissues. On the other hand, Bowen et al. [9] reported that the loss of NKX3.1 expression was strongly associated with hormone-refractory disease and advanced tumor stage in prostate cancer. In general, SDCs are typically positive for AR in immunohistochemical examination [4]; thus, the expression of NKX3.1 was estimated to be relevant to SDC tumorigenicity. The role of NKX3.1 gene expression during mouse salivary gland development has been reported by Schneider et al. [13]. In our study, NKX3.1 immunoreactivity was detected in PA (92.3\%) as well as in normal salivary glands, such as in the ducts and acini. The expression pattern of NKX3.1 in normal salivary gland or its lesions was similar to that in the prostate gland. However, NKX3.1 expression in SDC cases (35.7\%) was decreased compared with that in PA or in normal tissues. As SDC is a highgrade salivary gland malignancy, the lower immunohistochemical stain score for NKX3.1 is similar to that in previous reports showing loss of NKX3.1 expression in advanced prostate cancer $[9,14]$. As both SDC and prostatic adenocarcinoma express AR, SDC seems to share the AR-controlled pathway related to NKX3.1 with prostatic cancer in its tumorigenesis. However, in human prostatic lesions including hyperplasia and adenocarcinoma, the role of NKX3.1 in prostatic cancer is still controversial [9, $8,12,14,15]$. Further consideration is needed to yield any findings about the role of NKX3.1 in the prostate as well as in the salivary gland.

AMACR and PAP, which have been established as markers for prostate carcinoma, had positive detection rates of 58.5 and $33.3 \%$, respectively, in our SDC immunohistochemical results. AMACR, also known as P504S, plays a role in the beta-oxidation of branched-chain fatty acids and fatty acid derivatives [16]. Jiang et al. [17] reported that carcinomas arising from tissues that normally do not express AMACR, were also positive for AMACR, including $94 \%$ of prostate carcinomas, $31 \%$ of urothelial carcinomas, and $27 \%$ of gastric adenocarcinomas. Zhou et al. [18] reported that more than $60 \%$ of ovarian carcinomas and $30 \%$ of breast cancers and melanomas overexpress AMACR. Our results are consistent with these reports. AMACR is expressed in SDC, but not in the normal salivary gland. Thus, it is suggested that AMACR plays a pathogenic role in SDC. Additionally, PAP is a prostatic epithelium-specific differentiation antigen that has been implicated in the growth of prostatic carcinoma cells and this process is regulated by androgen $[19,20]$. Fan et al. [21] reported that $58.3 \%$ of SDCs were positive for PAP, whereas PSA was detected in $30 \%$ of SDCs. These findings might be related to the tumoral/neoplastic expression of AMACR or PAP in SDC tumorigenesis.

SDC is a clinically aggressive malignant tumor, but our results suggest that SDC ex PA has a better prognosis than de novo SDC. Significant prognostic factors in carcinoma ex PA include tumor stage, grade, proportion of carcinoma, extent of invasion, and proliferation index [22]. Generally, carcinoma ex PA has a relatively good prognosis, regardless of the carcinoma subtype, compared with that of other malignant tumors in salivary glands. We tested the utility of NKX3.1, AMACR, and PAP as prognostic factors for SDC, because the prognostic value of prostatic carcinoma markers in SDC has not been evaluated. Interestingly, AMACR and PAP immunoreactivity rates were higher in recurrence cases (local recurrence and distant metastasis) than in cases with no recurrence, especially for PAP. He et al. [23] showed that high expression of AMACR was significantly associated with an aggressive phenotype, with advanced tumor staging, increased vascular invasion, and increased perineural inva-
Takada/Nishida/Oyama/Kusaba/ Kadowaki/Arakane/Wada/Urabe/Daa 
sion in oral squamous cell carcinoma. Zha et al. [24] reported that AMACR is essential for prostatic cancer cell proliferation in vitro and that its enzymatic activity is elevated in prostatic cancer tissue. Xu et al. [25] reported that PAP-positive cases exhibited higher metastasis rates, larger tumors, more localized tumors, and higher pathological grades than did PAP-negative cases. Furthermore, the survival duration was also shorter in the PAP-positive group, as reported previously, suggesting that PAP has a prognostic value in prostate cancer [25]. Based on these reports, the immunohistochemistry of AMACR and PAP is possibly used as a prognosticator in SDC. Accordingly, SDC with local recurrence or distant metastasis showed higher rates of AMACR and PAP positivity. Thus, immunoreactivity of AMACR and PAP could be useful for assessing prognosis in SDCs. Although our clinical data indicate somewhat better prognosis in SDC ex PA than in de novo SDC, the immunohistochemical profile of the examined markers showed no significant difference. Thus, other factors may affect the difference in the biology of these two types of tumors.

Our results showed that SDC frequently expresses AR (81.0\%), NKX3.1 (35.7\%), and AMACR (58.5\%) and occasionally expresses PAP (33.3\%), indicating a close immunophenotypic homology with prostatic adenocarcinoma, except for PSA (0\%). With regard to clinical pathology, our data show that the immunohistochemical staining of prostate-specific markers should be interpreted with caution, in the appropriate morphological context, when determining whether a metastatic tumor is of prostate origin, especially when patients have a history of SDC. PSA and histopathological findings might be useful for distinguishing SDC from prostatic carcinoma.

The standard treatment for SDC is surgical resection with or without radiation therapy. The tumor is resistant to chemotherapy and the mortality rate is high [26]. Currently, there are only few treatment modalities available for recurrent and metastatic SDC in practice [27]. Recently, androgen deprivation therapy (ADT), a mainstay treatment for metastatic prostate cancer, has been used for the treatment of metastatic and recurrent AR-positive SDC [28-30]. Case series have reported a clinical benefit of ADT for recurrent and/or metastatic AR-positive salivary gland cancer; most of these cases were SDC or adenocarcinoma not otherwise specified [28-30]. Based on the resistance of SDC to chemotherapy, ADT might become a useful treatment option.

In conclusion, we revealed the immunohistochemical reaction of some prostate cancer markers for SDC. NKX3.1 expression is useful for SDC diagnosis. NKX3.1 expression is significantly decreased in SDC compared to PA; however, it was not correlated with SDC progression or advanced stage. The immunoreactivity of AMACR and PAP could be useful for assessing prognosis in SDCs, but with regard to pathology practice, immunohistochemical staining of prostate-specific markers should be interpreted with caution when determining whether a metastatic tumor is of prostate origin, especially when patients have a history of SDC.

\section{Statement of Ethics}

This study was approved by an ethics committee and institutional review board (number: 1637).

\section{Disclosure Statement}

The authors have no financial conflicts of interest.

\section{References}

1 Kikuchi Y, Hirota M, Iwai T, Aoki S, Chikumaru H, Kawabe R, et al. Salivary duct carcinoma in the mandible: a case report. Oral Surg Oral Med Oral Pathol Oral Radiol Endod. 2007 Mar;103(3):e41-6.

2 Barnes L, Eveson JW, Reichart P, Sidransky D, editors. World Health Organization Classification of Tumors. Pathology and Genetics of Head and Neck Tumors. Lyon: IARC Press; 2005.

3 Jaehne M, Roeser K, Jaekel T, Schepers JD, Albert N, Löning T. Clinical and immunohistologic typing of salivary duct carcinoma: a report of 50 cases. Cancer. 2005 Jun;103(12): 2526-33.

Immunomarker Expression in Salivary

Duct Carcinoma
4 Williams L, Thompson LD, Seethala RR, Weinreb I, Assaad AM, Tuluc M, et al. Salivary duct carcinoma: the predominance of apocrine morphology, prevalence of histologic variants, and androgen receptor expression. Am J Surg Pathol. 2015 May;39(5):705-13.

5 Feldman BJ, Feldman D. The development of androgen-independent prostate cancer. Nat Rev Cancer. 2001 Oct;1(1):34-45.

6 Ricke EA, Williams K, Lee YF, Couto S, Wang Y, Hayward SW, et al. Androgen hormone action in prostatic carcinogenesis: stromal androgen receptors mediate prostate cancer progression, malignant transformation and metastasis. Carcinogenesis. 2012 Jul;33(7):1391-8.
7 Abate-Shen C, Shen MM, Gelmann E. Integrating differentiation and cancer: the Nkx3.1 homeobox gene in prostate organogenesis and carcinogenesis. Differentiation. $2008 \mathrm{Jul}$; 76(6):717-27.

8 Aslan G, Irer B, Tuna B, Yorukoglu K, Saatcioglu F, Celebi I. Analysis of NKX3.1 expression in prostate cancer tissues and correlation with clinicopathologic features. Pathol Res Pract. 2006;202(2):93-8.

9 Bowen C, Bubendorf L, Voeller HJ, Slack R, Willi N, Sauter G, et al. Loss of NKX3.1 expression in human prostate cancers correlates with tumor progression. Cancer Res. 2000 Nov;60(21):6111-5. 
10 Bethel CR, Faith D, Li X, Guan B, Hicks JL, Lan F, et al. Decreased NKX3.1 protein expression in focal prostatic atrophy, prostatic intraepithelial neoplasia, and adenocarcinoma: association with Gleason score and chromosome 8p deletion. Cancer Res. 2006 Nov; 66(22):10683-90.

11 Tan PY, Chang CW, Chng KR, Wansa KD, Sung WK, Cheung E. Integration of regulatory networks by NKX3-1 promotes androgen-dependent prostate cancer survival. $\mathrm{Mol}$ Cell Biol. 2012 Jan;32(2):399-414.

12 Irer B, Toylu A, Aslan G, Celebi I, Yorukoglu K, Atabey N. Increased expression of NKX3.1 in benign prostatic hyperplasia. Urology. 2009 May;73(5):1140-4.

13 Schneider A, Brand T, Zweigerdt R, Arnold $\mathrm{H}$. Targeted disruption of the Nkx3.1 gene in mice results in morphogenetic defects of minor salivary glands: parallels to glandular duct morphogenesis in prostate. Mech Dev. 2000 Jul;95(1-2):163-74.

14 Korkmaz CG, Korkmaz KS, Manola J, Xi Z, Risberg B, Danielsen H, et al. Analysis of androgen regulated homeobox gene NKX3.1 during prostate carcinogenesis. J Urol. 2004 Sep;172(3):1134-9.

15 Ornstein DK, Cinquanta M, Weiler S, Duray PH, Emmert-Buck MR, Vocke CD, et al. Expression studies and mutational analysis of the androgen regulated homeobox gene NKX3.1 in benign and malignant prostate epithelium. J Urol. 2001 Apr;165(4):1329-34.

16 Ferdinandusse S, Denis S, IJlst L, Dacremont G, Waterham HR, Wanders RJ. Subcellular localization and physiological role of alphamethylacyl-CoA racemase. J Lipid Res. 2000 Nov;41(11):1890-6.
17 Jiang Z, Fanger GR, Woda BA, Banner BF, Algate $\mathrm{P}$, Dresser $\mathrm{K}$, et al. Expression of alphamethylacyl-CoA racemase (P504s) in various malignant neoplasms and normal tissues: a study of 761 cases. Hum Pathol. 2003 Aug; 34(8):792-6.

18 Zhou M, Chinnaiyan AM, Kleer CG, Lucas PC, Rubin MA. Alpha-Methylacyl-CoA racemase: a novel tumor marker over-expressed in several human cancers and their precursor lesions. Am J Surg Pathol. 2002 Jul;26(7):92631.

19 Lin MF, DaVolio J, Garcia-Arenas R. Expression of human prostatic acid phosphatase activity and the growth of prostate carcinoma cells. Cancer Res. 1992 Sep;52(17):4600-7.

20 Meng TC, Lin MF. Tyrosine phosphorylation of c-ErbB-2 is regulated by the cellular form of prostatic acid phosphatase in human prostate cancer cells. J Biol Chem. 1998 Aug; 273(34):22096-104.

21 Fan CY, Wang J, Barnes EL. Expression of androgen receptor and prostatic specific markers in salivary duct carcinoma: an immunohistochemical analysis of 13 cases and review of the literature. Am J Surg Pathol. 2000 Apr; 24(4):579-86.

22 Lewis JE, Olsen KD, Sebo TJ. Carcinoma ex pleomorphic adenoma: pathologic analysis of 73 cases. Hum Pathol. 2001 Jun;32(6):596604.

23 He HL, Lee YE, Chang MT, Shiue YL, Chang SL, Chen TJ, et al. AMACR overexpression acts as a negative prognostic factor in oral squamous cell carcinoma. Int J Med Sci. 2018 Apr;15(6):638-44.
24 Zha S, Ferdinandusse S, Denis S, Wanders RJ, Ewing CM, Luo J, et al. Alpha-methylacyl$\mathrm{CoA}$ racemase as an androgen-independent growth modifier in prostate cancer. Cancer Res. 2003 Nov;63(21):7365-76.

25 Xu H, Wang F, Li H, Ji J, Cao Z, Lyu J, et al. Prostatic acid phosphatase (PAP) predicts prostate cancer progress in a populationbased study: the renewal of PAP? Dis Markers. 2019 Jan;2019:7090545.

26 Dalin MG, Desrichard A, Katabi N, Makarov V, Walsh LA, Lee KW, et al. Comprehensive molecular characterization of salivary duct carcinoma reveals actionable targets and similarity to apocrine breast cancer. Clin Cancer Res. 2016 Sep;22(18):4623-33.

27 Limaye SA, Posner MR, Krane JF, Fonfria M, Lorch JH, Dillon DA, et al. Trastuzumab for the treatment of salivary duct carcinoma. Oncologist. 2013;18(3):294-300.

28 Locati LD, Perrone F, Cortelazzi B, Imbimbo M, Bossi P, Potepan P, et al. Activity of abiraterone in rechallenging two AR-expressing salivary gland adenocarcinomas, resistant to androgen-deprivation therapy. Cancer Biol Ther. 2014 Jun;15(6):678-82.

29 Locati LD, Perrone F, Cortelazzi B, Lo Vullo S, Bossi P, Dagrada G, et al. Clinical activity of androgen deprivation therapy in patients with metastatic/relapsed androgen receptorpositive salivary gland cancers. Head Neck. 2016 May;38(5):724-31.

30 Yamamoto N, Minami S, Fujii M. Clinicopathologic study of salivary duct carcinoma and the efficacy of androgen deprivation therapy. Am J Otolaryngol. 2014 Nov-Dec;35(6): 731-5. 